\title{
RETRACTED ARTICLE: Cochlear implant device activation after 7 days in cochlear implant recipients
}

\author{
Mingfang Diao $^{1}\left(\mathbb{D} \cdot\right.$ Jianjun Sun ${ }^{1} \cdot$ Fangjie Tian $^{1} \cdot$ Yili Ding ${ }^{1} \cdot$ Yuqing Wang ${ }^{1}$
}

Received: 29 June 2018 / Accepted: 11 September 2018 / Published online: 17 September 2018

๑) Springer-Verlag GmbH Germany, part of Springer Nature 2018

The authors have retracted this article because it contains sections that substantially overlap with the following articles (amongst others) [1,2]. All authors have agreed to this retraction. The online version of this article contains the full text of the retracted article as electronic supplementary material.

1. Alsabellha, RM., Hagr A, Al-momani MO, and Garadat SN "Cochlear Implant Device Activation and
Programming : 5 Days Postimplantation", Otology \& Neurotology. 35(4):e130-e134, APR 2014

2. Abdulrahman Hagr, Soha N. Garadat, Murad Al-Momani, Rabea M. Alsabellha \& Fida A. Almuhawas (2015) Feasibility of one-day activation in cochlear implant recipients, International Journal of Audiology, 54:5, 323-328, https:// doi.org/10.3109/14992027.2014.996824

Electronic supplementary material The online version of this article (https://doi.org/10.1007/s00405-018-5129-7) contains supplementary material, which is available to authorized users.

Mingfang Diao

diaomingfang@yeah.net

1 Center of Otolaryngology of PLA, Navy General Hospital,

Fucheng Road 6, Haidian District, Beijing 100048,

People's Republic of China 